\title{
Philosophiques
}

\section{Bonnette, Lucie, Le fondement religieux de la pensée de Jung,}

Montréal, Fides, 1986, III pp.

\section{René F. Marineau}

Volume 14, numéro 2, automne 1987

URI : https://id.erudit.org/iderudit/027022ar

DOI : https://doi.org/10.7202/027022ar

Aller au sommaire du numéro

Éditeur(s)

Société de philosophie du Québec

ISSN

0316-2923 (imprimé)

1492-1391 (numérique)

Découvrir la revue

Citer ce compte rendu

Marineau, R. F. (1987). Compte rendu de [Bonnette, Lucie, Le fondement religieux de la pensée de Jung, Montréal, Fides, 1986, III pp.] Philosophiques,

14(2), 435-437. https://doi.org/10.7202/027022ar d'utilisation que vous pouvez consulter en ligne.

https://apropos.erudit.org/fr/usagers/politique-dutilisation/ 


\title{
COMPTES RENDUS
}

BONNETTE, Lucie, Le fondement religieux de la pensée de Jung, Montréal, Fides, 1986, III pp.

\author{
par René F. Marineau
}

L'œuvre de Carl Gustav Jung a connu, au cours des dernières années, un renouveau de curiosité. Plusieurs raisons expliquent ce phénomène: parmi celles-ci, il faut citer l'intérêt grandissant pour la psychologie dite transpersonnelle. Cet intérêt n'est ni étranger à un retour des valeurs dites «spirituelles », ni coupé du phénomène du renouveau religieux dans la société.

C'est dans cette perspective qu'il faut accueillir le livre de Lucie Bonnette. L'auteure propose une lecture de Jung à partir du fondement religieux de la pensée de celui-ci. Cela me semble légitime, indispensable même : on ne peut comprendre et apprécier Jung à moins de retourner aux assises mêmes de son interprétation de la vie et de l'univers. Selon que l'on est en accord ou pas avec Jung, cela permettra ici, de recadrer sa pensée ou là, de démasquer son véritable propos. Dans tous les cas, la lecture du livre de madame Bonnette nous interpelle au niveau d'un nécessaire exorcisme de nos préjugés philosophiques et scientifiques à l'endroit de Jung.

Le premier chapitre traite de la vie et l'œuvre de Jung sous l'angle du développement de sa pensée religieuse : l'auteure aborde à grands traits les éléments qui lui apparaissent expliquer la pensée religieuse de Jung. Dans un premier temps la relation de Freud et Jung : cette partie pose problème, car je comprends mal qu'elle soit présentée en tout début et de manière aussi superficielle. Si elle a une telle importance, il est nécessaire de lui accorder non seulement la première place, mais aussi l'approfondissement souhaité : nous n'avons malheureusement droit qu'à quelques paragraphes qui n'éclairent pas vraiment la thèse fondamentale des différences profondes entre les deux hommes. Suit une partie sur l'origine de la pensée religieuse de Jung. L'auteure présente une séquence de rêves, fantasmes et événements qui semblent avoir une cohérence certaine, mais qui prête à tellement d'interprétations différentes : il faut alors accepter la signification proposée comme un dogme, dogme qui 
alimentera une compréhension qui deviendra alors toute logique. Le chapitre se termine sur la présentation d'auteurs qui ont influencé la pensée religieuse et clinique de Jung : liste partiale et partielle, où chaque auteur est présenté en trois ou quatre lignes. Le tout donne l'impression d'un essai d'interprétation biographique à rebours et à rabais: l'auteure ne fait justice à personne, certainement pas à Jung, à Freud, à Bergson ou Janet; encore moins à ellemême.

Le second chapitre, étude synthétique et critique de la pensée jungienne, est beaucoup mieux. L'auteure reste plus près de Jung, ce qui lui facilite les choses, certes, mais permet aussi au lecteur de mieux se centrer sur le propos fondamental du livre. La thèse à étayer ici est que la psychologie religieuse de Jung n'est pas un domaine d'intérêt particulier pour le psychiatre suisse, mais représente l'essentiel de sa pensée sur l'être humain. Cette thèse est bien présentée, avec clarté et concision. Ce chapitre permet de découvrir le vrai Jung, celui qui diffère radicalement de Freud, celui dont les a priori conditionnent la conception qu'il se fait de la personne humaine, de la psychothérapie et de la science : c'est ici que les choix de valeurs de Jung sont clairs et impliquants. Et je dis bien choix de valeurs et non démonstrations scientifiques.

Le troisième chapitre traite de la psychothérapie jungienne. L'auteure présente les particularités de l'intervention jungienne qui se résument finalement par l'ajout par le psychiatre suisse du plan de la métamorphose et de la thérapeutique du midi ; quant au reste, on y retrouve l'essentiel de Freud, sous le couvert d'un nouveau vocabulaire. Le processus d'individuation dont la résultante est la réconciliation du conscient et de l'inconscient est l'objectif thérapeutique et éducatif ultime et s'opère à travers l'expérience religieuse.

I.e quatrième chapitre apporte peu de choses nouvelles, sinon que Jung $y$ est décrit comme un clinicien et un scientifique : comme clinicien il est d'abord un «spécialiste de la psychopathologie et un homme pour qui les faits sont plus importants que les théories »; comme scientifique, il adopte le processus descriptif et aboutit ainsi à la mise à jour et à la vérification de ses hypothèses. Cela l'apparente à la phénoménologie et démarque son cheminement scientifique. On part pourtant d'un a priori. Jung a combattu toute sa vie la "psychologie sans âme » et a inscrit sur le fronton de sa maison: "Appelé ou non appelé, Dieu sera présent ».

Le livre de Lucie Bonnette m'apparaît très intéressant à divers points de vue. Dans l'ensemble, il est écrit simplement et l'auteure réussit à expliquer des notions jungiennes ordinairement imprécises et difficiles. Il y a, ici et là, des longueurs et des redites. Mais Jung ètant lui-même si compliqué et complexe à déchiffrer, il faut en savoir gré à une personne qui réussit à le traduire avec une aise relative.

Sur le fond, l'auteure aborde l'étude de Jung par « le bon bout », soit celui du fondement religieux de la pensée du médecin zurichois. Il est juste de dire que l'on ne peut comprendre Jung si on ne retourne pas à la base de sa pensée thérapeutique et scientifique. Bien plus, on ne peut prétendre être radicalement 
jungien, à moins d'adhérer à ce dogme qui veut que " chaque être humain est à la recherche de son âme». Cela l'auteure sait le dire et nous en convaincre.

Ce livre repose le problème fondamental de l'héritage de Jung. Au plan épistémologique, les bases sur lesquelles reposent le "système" jungien me semblent bien fragiles. Cela n'ébranlera pas les inconditionnels : les disciples ne sont jamais à court de rationalisations et de preuves scientifiques irréfutables. Ils trouveront, dans le livre de Lucie Bonnette, riche matière à réflexion et à confort. Les lecteurs plus immédiatement désintéressés regretteront l'absence de critique chez Lucie Bonnette. Mais les faiblesses du système jungien et la quasi-absence de critique de l'auteure ici ne sont pas exception : l'aveuglement n'est pas l'apanage d'un seul système.

Cette limite chez l'auteure ne devrait pas empêcher une lecture de son livre et des œuvres de Jung lui-même. Certes on peut prendre tout de Jung ou le rejeter en bloc. Il est possible, aussi, de puiser chez lui des éléments fort valables, au niveau surtout des son intuition clinique, de la compréhension du monde de la psychose et de l'inépuisable source de créativité chez la personne humaine.

Le livre de Lucie Bonnette est valable et recommandable pour diverses catégories de lecteurs : les jungiens, les anti-jungiens et le public plus large qui veut connaître, approfondir, se faire une idée... Il ne remplace en rien les écrits de Jung lui-même, écrits, qui en dépit de difficultés et controverses certaines, demeurent riches et stimulants.

Département de psychologie, Université du Québec à Trois-Rivières 